\title{
Experimental and Theoretical Deflections of Hybrid Composite Sandwich Panel under Four-point Bending Load
}

\author{
Fajrin, J.*, Zhuge, Y.2, Wang, H.², and Bullen, F.2
}

\begin{abstract}
This paper presents a comparison of theoretical and experimental deflection of a hybrid sandwich panel under four-point bending load. The paper initially presents few basic equations developed under three-point load, followed by development of model under four-point bending load and a comparative analysis between theoretical and experimental results. It was found that the proposed model for predicting the deflection of hybrid sandwich panels provided fair agreement with the experimental values. Most of the sandwich panels showed theoretical deflection values higher than the experimental values, which is desirable in the design. It was also noticed that the introduction of intermediate layer does not contribute much to reduce the deflection of sandwich panel as the main contributor for the total deflection was the shear deformation of the core that mostly determined by the geometric of the samples and the thickness of the core.
\end{abstract}

Keywords: Deflection; four-point bending; hybrid sandwich panel; theoretical model.

\section{Introduction}

A concept of hybrid sandwich structure with intermediate layer has been introduced by Mamalis et al. [1] which significantly enhanced the load carrying capacity of the original sandwich panel. An experimental investigation with different wood based materials for the intermediate layer in smallscale samples was conducted by Fajrin et al. [2] and the results confirmed the claim of the previous study [1]. A further investigation with statistical design of experiment approach has also confirmed the significant impovement achieved by the introduction of the intermediate layer [3]. It was found that intermediate layer made of jute fiber composite and medium density fiber improved the structural performance of the hybrid sandwich panel.

The basic theoretical concept of hybrid sandwich panel with intermediate layer that established under three-point bending load scheme has been discussed throughly as reported by Fajrin [4].

\footnotetext{
${ }^{1}$ Department of Civil Engineering, Faculty of Engineering, University of Mataram, Mataram 83115, Nusa Tenggara Barat, INDONESIA

2 Centre of Excellence in Engineered Fibre Composites (CEEFC), University of Southern Queensland (USQ), Toowoomba, QLD 4350, AUSTRALIA

*Corresponding author; e-mail: jauhar.fajrin@unram.ac.id
}

Note: Discussion is expected before June, $1^{\text {st }} 2017$, and will be published in the "Civil Engineering Dimension", volume 19, number 2, September 2017.

Received 07 October 2016; revised 06 January 2017; accepted 24 February 2017.
The established model showed the potential of the concept to improve the flexural rigidity and stiffness of the sandwich panel. The hybrid sandwich panel containing intermediate layer with intermediate properties between the skins and core, generates a higher stiffness by only a slight increase in weight contributed by intermediate layer. More recently, the behaviour of the new developed panel under in-plane shear loading has been reported [5], which specifically focused on statistical analysis of the results. In the real application, however, it is also important to evaluate the performance of a structure under a pure bending load scheme (four-point bending load). This paper discusses the development of theoretical model of hybrid sandwich panel with intermediate layer under four-point bending load. The comparison of theoretical and experimental deflection were provided. This paper presents few basic equations developed under three-point load, followed by the development of model under four-point load and a comparative analysis between theoretical and experimental results.

\section{Basic Concept of the New Developed Hybrid Composite Sandwich Panel}

The research work focuses on introducing a new layer in between the skin and the core of a standard sandwich panel structure to form a hybrid structure. A natural fiber composites (NFC) laminate is placed as an intermediate layer in between aluminum skin and expanded polystyrene (EPS) core to produce a hybrid composite sandwich panel as shown in Figure 1(b). Hence, this new structure is a combination of two components; a panel with aluminum skins and EPS core as an integrated sandwich structure and 
intermediate layer laminates made of NFC that resulting in a hybrid structure. When a monolithic panel manufactured as a homogeneous material is subjected to a loading scheme, the typical stress distribution is a straight diagonal line from the top to the bottom surface as shown by the dotted-line in Figure 1. The stress distribution, however, will have a considerable transformation at the top and bottom interface between the skin and core layers for sandwich structure, as shown in Figure 1(a). Many authors have identified the stress discontinuity as a prime contributor for failure in sandwich panel. The idea of introducing an intermediate layer, which has intermediate properties between the skins and core, is to reduce the problem, as depicted in Figure 1(b).

This concept can be best explained using the Hooke's laws which relate induced stress to the material's modulus of elasticity. When intermediate layers with elastic modulus between those of the skin and core are inserted, the abrupt step between the high and low stresses within the skins and core can be reduced. This configuration, of two layers of skins and intermediate layers and the core in between, theoretically generates a higher flexural strength for the sandwich panel. Davies [6] highlighted that in sandwich construction, the yield stress of skin material is of less concern because the load carrying capacity of the structure is typically determined by wrinkling of the face in compression or by the shear failure of the core. It is thus becomes crucial to provide more lateral support for the face by introducing another layer between those of the faces and core. The current common approach to address the issue is either increases the faces thickness or improves the quality of the core. Both approaches however may have significant impact on the overall cost. The price of skin material is normally expensive, while the price of the core is much less than that of the skins, but an increased thickness of the core can also result in higher overall cost.

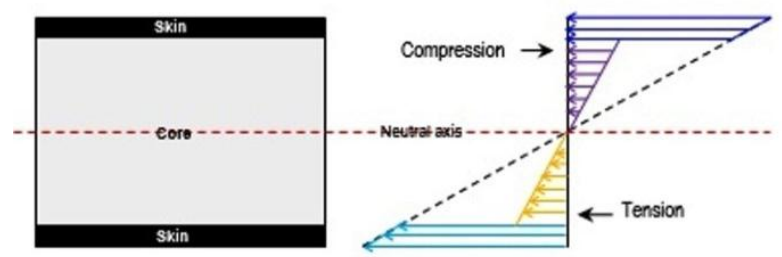

(a) Conventional sandwich panel

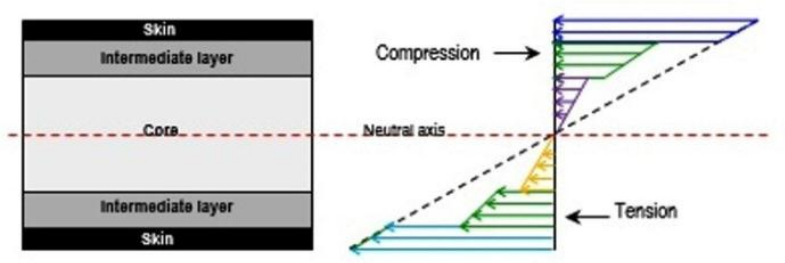

(b) Hybrid sandwich panel

Figure 1. Typical Stress Distribution in Sandwich Panel: (a) Conventional Sandwich Panel and (b) Hybrid Sandwich Panel with Intermediate Layer

\section{Development of Theoretical Frameworks for Deflection under Four-Point Bending Load}

In a homogeneous material, the deflection due to shear is often neglected. For a sandwich panel, however, the core material is usually not rigid in shear and thus the deflection is not negligible in most cases [7]. The deflection, $\delta$, of a homogeneous beam under three-point bending load is given by:

$\delta=\frac{\mathrm{FL}^{3}}{48 \mathrm{EI}}$

In which $\mathrm{F}$ is the applied load and $\mathrm{L}$ is the span length, while EI is the flexural rigidity which is the product of the modulus of elasticity (E) and the second moment of area (I). In the case of sandwich panel, this equation can be modified as [8]:

$$
\delta=\frac{\mathrm{FL}^{3}}{48(\mathrm{EI})_{\mathrm{eq}}}
$$

The contribution of the core shear to the deflection of sandwich structure that is commonly neglected in ordinary beam analysis can be obtained as per Equation 3 [8].

$$
\delta=\frac{\mathrm{FL}}{4(\mathrm{AG})_{\mathrm{eq}}}
$$

Where $A$ is the area and $G$ is the shear modulus of the core material. The total deflection at the center point of sandwich beam can be obtained by Equation 4:

$$
\delta=\frac{\mathrm{FL}^{3}}{48(\mathrm{EI})_{\mathrm{eq}}}+\frac{\mathrm{FL}}{4(\mathrm{AG})_{\mathrm{eq}}}
$$

For the flexural properties of sandwich structure under four-point bending load, ASTM C 393-00 [9] stated that the total deflection is a sum of deflection due to bending and shear as shown in Equation 5, where $\mathrm{D}\left(\mathrm{N}-\mathrm{mm}^{2}\right)$ is the stiffness, $\mathrm{U}(\mathrm{N})$ is the panel shear rigidity, $\mathrm{P}(\mathrm{N})$ is the applied load and $\mathrm{L}(\mathrm{mm})$ is the span length.

$$
\delta=\frac{11 \mathrm{PL}^{3}}{768 \mathrm{D}}+\frac{\mathrm{PL}}{8 \mathrm{U}}
$$

The above equation is derived from a flexural test under four-point bending load at quarter point loading span, as shown in Figure 2 (a). Under a fourpoint bending load with third point loading scheme in which the two point loads applied at an equal distance $(\mathrm{L} / 3)$ of the span length, as depicted in Figure 2(b), Roylance [10] recommended the following equation: 


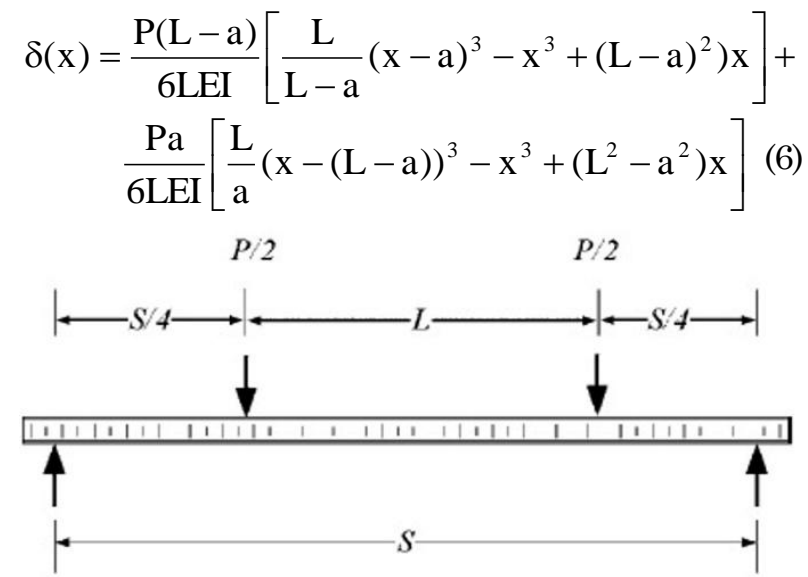

(a) Quarter point loading

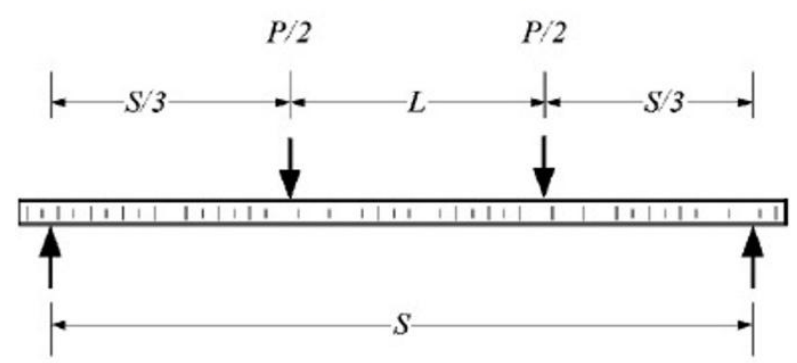

(b) Third point loading

Figure 2. Two Alternatives for Applying Load in Fourpoint Bending Load Scheme as Described in ASTM C 39300 [9]. (a) Quarter Point Loading Scheme, and (b) Third Point Loading Scheme.

For the third load scheme, equal load distance was applied, with $\mathrm{a}=\mathrm{L} / 3$ and $\mathrm{x}=\mathrm{L} / 2$. Including these two values in the above equation results in:

$\delta=\frac{23 \mathrm{PL}^{3}}{1296 \mathrm{EI}}$

For sandwich panel, this equation can be modified as

$\delta=\frac{23 \mathrm{PL}^{3}}{1296(\mathrm{EI})_{\mathrm{eq}}}$

As mentioned earlier, the contribution of shear deflection should be considered in sandwich panel especially when a low density core is employed. The deformation under four-point bending load is shown in Figure 3. The deflection of the loading point due to this deformation mode is given by the following equations $[4,8]$ :

$\frac{\delta}{\mathrm{L} / 3}=\gamma=\frac{\mathrm{Q}}{\mathrm{G}_{\mathrm{c}} \mathrm{bd}}$

$\delta=\frac{\mathrm{Q}(\mathrm{L} / 3)}{\mathrm{G}_{\mathrm{c}} \mathrm{bd}}$

Since in third point bending load, $\mathrm{Q}=\mathrm{P} / 2$, then

$\delta=\frac{\mathrm{PL}}{6(\mathrm{AG})_{\mathrm{eq}}}$

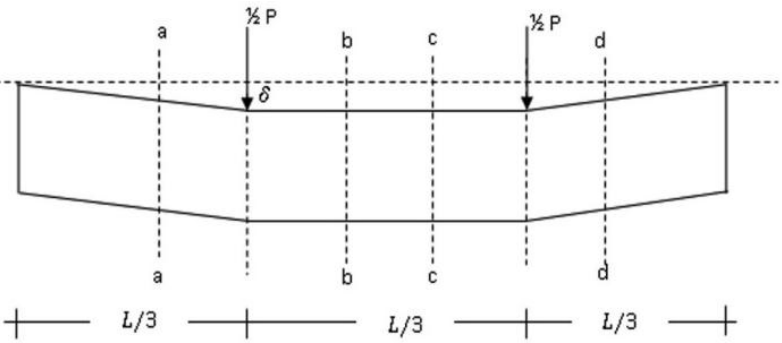

Figure 3. Deformation due to Core Shear under Fourpoint Loading Scheme [4, 8].

Hence, the total deflection under four-point bending load is a linear superposition of the deflection due to bending and shear, which gives:

$\delta=\frac{23 \mathrm{PL}^{3}}{1296(\mathrm{EI})_{\mathrm{eq}}}+\frac{\mathrm{PL}}{6(\mathrm{AG})_{\mathrm{eq}}}$

The above equation confirms the equation derived by Manalo et al. [11] for sandwich panel beam in flatwise position:

$\Delta=\frac{23 \mathrm{PL}^{3}}{1296 \mathrm{D}}+\frac{\mathrm{PL}}{6 \mathrm{AG}}$

In which $\mathrm{D}$ is the equivalent flexural rigidity. Determining shear modulus of core material $\left(\mathrm{G}_{c}\right)$ experimentally is technically difficult. Somayaji [12] suggested the following relationship as a convenient procedure to establish such value:

$\mathrm{G}_{\mathrm{c}}=\frac{\mathrm{E}}{2(1+\mathrm{v})}$

For conventional sandwich panel, $(\mathrm{EI})_{\text {eq }}$ is defined by Equation $15[7,8]$.

$(E I)_{e q}=E_{f} \frac{b t^{3}}{6}+E_{f} \frac{b t d^{2}}{2}+E_{c} \frac{b c^{3}}{12}$

Where $t$ is the thickness of the face, $b$ is the panel width, $d$ is the distance between the midplanes of the upper and bottom faces and $\mathrm{c}$ is the core thickness. $\mathrm{E}_{\mathrm{f}}$ and $\mathrm{E}_{\mathrm{c}}$ are the elastic modulus of the skins and core respectively.

While for hybrid sandwich panels with intermediate layer, it is calculated based on Equation 16 as proposed by Fajrin [4].

$$
(E I)_{e q}=E_{f}\left[\frac{b t_{f}^{3}}{6}+\frac{b t_{f} d_{1}^{2}}{2}\right]+E_{i}\left[\frac{b t_{i}^{3}}{6}+\frac{b t_{i} d_{2}^{2}}{2}\right]+E_{c} \frac{b t_{c}^{3}}{12}
$$

Where $d_{1}$ and $d_{2}$ are the distance between the midplanes of the upper and bottom faces and intermediate layer respectively. The thicknesses of materials; face, intermediate layer and core are denoted as $t_{f}, t_{i}$ and $t_{c}$ respectively. $E_{f}, E_{i}$ and $E_{c}$ are 
the modulus elasticity of constituent materials; face, intermediate layer and core respectively.

\section{Sample Preparations and Experimental Program}

In order to verify the developed model, two stages of experimental work with medium and large scale samples were conducted subsequently. For medium scale; two groups of hybrid sandwich panels with jute fibre composites (JFC) and hemp fibre composites (HFC) intermediate layer were compared to a group of samples without intermediate layer as the control (CTR). The thickness of the intermediate layer was maintained at $3 \mathrm{~mm}$. Each group of samples was replicated 5 times so that a total of 15 samples were tested in medium scale. The length of span was $450 \mathrm{~mm}$ and the samples were cut into the size of $550 \times 50 \times 22 \mathrm{~mm}$ for length, width and thickness, respectively. An aluminium sheet was cut into the required size for the skins, with the thickness of $0.5 \mathrm{~mm}$ for all samples. While expanded polystyrene (EPS) was employed for the core with a thickness of $21 \mathrm{~mm}$ for CTR specimens and $15 \mathrm{~mm}$ for hybrid sandwich panels (JFC and HFC). All medium scale samples were maintained to have a constant overall thickness of $22 \mathrm{~mm}$.

A similar arrangement was also used for the large scale experiment; three specimens groups which include two groups of hybrid sandwich panels and a group of conventional sandwich panels as the control. The large scale samples were shaped in the size of $1150 \times 100 \times 52 \mathrm{~mm}$ with the span length of 900 $\mathrm{mm}$. Jute fiber composites (JFC) and medium density fiber (MDF) were used for the intermediate layer of large scale samples with the thickness of 5 $\mathrm{mm}$. Similarly, the core of sandwich panel was an EPS foam with a thickness of $50 \mathrm{~mm}$ for CTR samples and $40 \mathrm{~mm}$ for hybrid panels (JFC and MDF). Aluminium sheet with a thickness of $1 \mathrm{~mm}$ was employed as the face of all samples in large scale experiment. A total of 15 samples that comprises of 5 samples for each group were tested in the large scale experiment.

The flexural testing was carried out using a $100 \mathrm{kN}$ servo-hydraulic machine with a loading rate of 5 $\mathrm{mm} / \mathrm{min}$. The longitudinal strains were measured using strain gauges attached at the middle top and bottom surface of samples. A system 5000 data logger recorded all the applied load, deflection and strains. The testing process were started by setting the loading pins to nearly touch the top surface of the specimen and terminated after a visible collapse mechanism was encountered. The testing was also terminated when the specimen undergoing large displacement without any increase in load. The actual set up of flexural testing is presented in Figure 4.

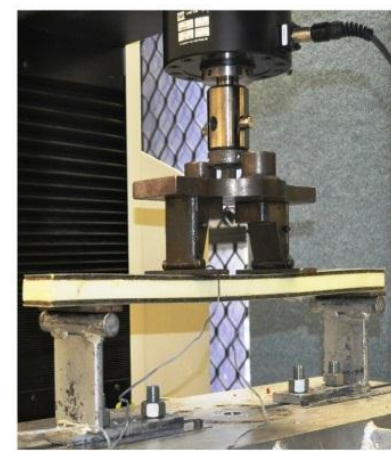

(a) Medium scale

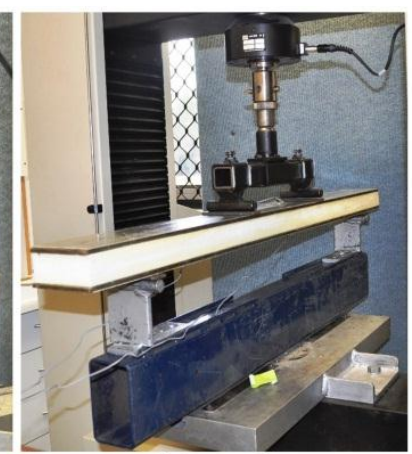

(b) Large scale
Figure 4. The Actual Set-up of Testing under Four-point Bending Load. (a) Testing Set-up for Medium Scale Samples, and (b) Testing Set-up for Large Scale Samples.

\section{Results and Discussion}

The theoretical values of deflection were estimated as per Equation 12 with the corresponding equivalent bending stiffness, (EI) $)_{\text {eq, }}$ for conventional and hybrid sandwich panels. The bending stiffness of conventional sandwich panel is calculated as per Equation 15, while for hybrid sandwich panels, it is calculated based on Equation 16. The results are presented in Table 1 and Table 2 . In order to simplify the analysis, only two samples were selected for each group to be discussed throughly. For the same reason, the analysis was only focused to compare the deflection of sandwich panels under two particular loads $(\mathrm{P})$, which is $50 \mathrm{~N}$ and $100 \mathrm{~N}$.

\section{Medium Scale Samples}

Table 1 presents the theoretical deflection $\left(\delta_{\text {theo }}\right)$ and experimental deflection $\left(\delta_{\exp }\right)$ values of medium scale sandwich panels in the linear elastic region. As mentioned earlier, two particular loads $(\mathrm{P})$ have been chosen in the elastic region of the load-deflection curve, which are $50 \mathrm{~N}$ and $100 \mathrm{~N}$ for the comparison purposes. In general, the experimental values were in reasonable agreement with the theoretical values. The differences range from $3.9 \%$ to $35.4 \%$. Most of the sandwich panels showed experimental values lower than the theoretical values, which according to Teles et al. [13] can be considered as highly desirable in the design. It can also be seen that for the selected samples in CTR group (CTR-1 and CTR-3), the difference between theoretical and experimental values ranges from $17.1 \%$ to $35.4 \%$. While the selected samples in JFC group (JFC-3 and JFC-5), the theoretical framework tended to underestimate the experimental deflection values. The theoretical estimation were approximately 4\%-33.1\% lower than the experimental values. Meanwhile, the theoretical deflection values for sandwich panel in HFC group 
Table 1. Theoretical and Experimental Deflection Values of Medium Scale Sandwich Panels

\begin{tabular}{|c|c|c|c|c|c|c|c|c|c|c|c|}
\hline \multirow{2}{*}{\multicolumn{2}{|c|}{ Samples }} & \multicolumn{2}{|c|}{ Geometric } & \multirow[b]{2}{*}{$(\mathrm{EI})_{\mathrm{eq}}$} & \multirow[b]{2}{*}{$\mathrm{P}$} & \multirow[b]{2}{*}{$\delta_{\mathrm{b}}$} & \multirow[b]{2}{*}{$\delta_{\mathrm{s}}$} & \multirow[b]{2}{*}{$\delta_{\text {theo }}$} & \multirow[b]{2}{*}{$\delta_{\exp }$} & \multirow{2}{*}{$\frac{\delta_{\text {theo }}}{\delta_{\exp }}$} & \multirow[b]{2}{*}{$\%$} \\
\hline & & $\mathrm{b}$ & $\mathrm{t}_{\mathrm{c}}$ & & & & & & & & \\
\hline \multirow{4}{*}{ CTR } & \multirow{2}{*}{1} & 51.57 & 22.80 & 478993062 & 50 & 0.17 & 1.18 & 1.35 & 1.0 & 1.35 & 35.4 \\
\hline & & 51.57 & 22.80 & 478993062 & 100 & 0.34 & 2.37 & 2.71 & 2.0 & 1.35 & 35.4 \\
\hline & \multirow{2}{*}{3} & 51.30 & 22.20 & 451118121 & 50 & 0.18 & 1.23 & 1.41 & 1.2 & 1.17 & 17.1 \\
\hline & & 51.30 & 22.20 & 451118121 & 100 & 0.36 & 2.45 & 2.81 & 2.3 & 1.22 & 22.2 \\
\hline \multirow{4}{*}{ JFC } & \multirow{2}{*}{3} & 50.30 & 15.03 & 509155453 & 50 & 0.16 & 1.85 & 2.01 & 2.1 & 0.96 & -4.5 \\
\hline & & 50.30 & 15.03 & 509155453 & 100 & 0.32 & 3.69 & 4.01 & 6.0 & 0.67 & -33.1 \\
\hline & \multirow{2}{*}{5} & 50,00 & 15.05 & 507097313 & 50 & 0.16 & 1.86 & 2.02 & 2.1 & 0.96 & -4.0 \\
\hline & & 50,00 & 15.05 & 507097313 & 100 & 0.32 & 3.71 & 4.03 & 6.0 & 0.67 & -32.8 \\
\hline \multirow{4}{*}{$\mathrm{HFC}$} & \multirow{2}{*}{1} & 50.50 & 16.55 & 546612658 & 50 & 0.15 & 1.67 & 1.82 & 1.9 & 0.96 & -4.3 \\
\hline & & 50.50 & 16.55 & 546612658 & 100 & 0.30 & 3.34 & 3.64 & 3.5 & 1.04 & 3.9 \\
\hline & \multirow{2}{*}{5} & 52.50 & 17.20 & 601680685 & 50 & 0.13 & 1.55 & 1.68 & 1.4 & 1.20 & 20.1 \\
\hline & & 52.50 & 17.22 & 601680685 & 100 & 0.27 & 3.09 & 3.36 & 3.0 & 1.12 & 12.1 \\
\hline
\end{tabular}

(HFC-1 and HFC-5) were higher than the experimental values, except for specimen HFC-1 under $50 \mathrm{~N}$ load. The difference within the HFC group ranges from $3.9 \%$ to $20.1 \%$.

Table 1 also shows the geometrics of samples; width (b) and core thickness ( $\left.t_{c}\right)$. The equivalent bending stiffness, $(\mathrm{EI})_{\mathrm{eq}}$, of both conventional and hybrid sandwich panels is also presented within the table, as well as the deflection due to bending $\left(\delta_{\mathrm{b}}\right)$ and the deflection due to shear $\left(\delta_{\mathrm{s}}\right)$. It is clearly shown that the hybrid sandwich panels provide reasonably higher equivalent bending stiffness. The result is certainly not surprising since the hybrid sandwich panels embedded an intermediate layer which was considered when estimating the equivalent bending stiffness. Theoretically, when bending stiffness increased the deflection should be decreased. However, it is not always the case in sandwich panels as shown in Table 1. Under the same load, the deflection of hybrid sandwich panel even higher than those of conventional sandwich panels. For example, the sandwich panels with JFC and HFC intermediate layer have the theoretical deflection values of $2.02 \mathrm{~mm}$ (JFC-5) and $1.82 \mathrm{~mm}$ (HFC-1), respectively. While conventional sandwich panel without intermediate layer (CTR) has a theoretical deflection value of $1.35 \mathrm{~mm}$ (CTR-1). The reason for this can be clearly obtained by checking the contribution of bending and shear deformation of the core to the overall deflection. As seen in Table 1, bending only contributes around $8 \%$ to $13 \%$ to the total deflection. For instance, the deflection due to bending for specimen CTR-3 under $100 \mathrm{~N}$ load was $0.36 \mathrm{~mm}$, which was only $12.81 \%$ of the overall deflection of $2.81 \mathrm{~mm}$. On the other hand, shear deformation contributes $87.19 \%$ to the total deflection $(2.45 \mathrm{~mm})$. Overall, the shear deformation of the core, which has very low shear modulus $\left(\mathrm{G}_{\mathrm{c}}=2.69\right.$ $\mathrm{MPa}$ ), was the main contributor for the overall theoretical deflection, approximately $87 \%$ to $92 \%$. The result confirms the finding reported by Sharaf et al. [14] which stated that the shear deformation is the significant contributor for the overall deflection of sandwich panels with soft core. More specifically, they reported that the contribution of shear deformation to the overall deflection is about $75 \%$ for sandwich panel with soft core and approximately $50 \%$ for hard core [14].

It seems that for deflection due to shear deformation of the core, as per Equation 11, the contribution of samples geometric, the width and the thickness of the core, is crucial. As seen in Table 1, the thicknesses of CTR samples' core $\left(t_{c}\right)$ were substantially higher than those of hybrid sandwich panel with JFC and HFC intermediate layer that resulting in smaller deflection. The thicknesses of the core for CTR group in medium scale samples showed in the table were around 22.2-22.8 $\mathrm{mm}$. While the core thicknesses of hybrid sandwich panels were measured around 15.3 to $17.2 \mathrm{~mm}$.

\section{Large Scale Samples}

The theoretical and experimental deflection values of the larger scale sandwich panels were presented in Table 2. Similar to the values for medium scale samples, there is reasonably agreement between the theoretical and experimental findings that range from $3.7 \%$ to $26 \%$ within the elastic region of loaddeflection curves.

For the large samples, it was observed that the experimental values were mostly lower than the theoretical values. The theoretical deflection values for sandwich panels with JFC intermediate layer are, however, lower than the experimental values. For the control group, the difference was approximately $8.9 \%$ to $26 \%$. Within JFC group, the values differ by $6.2 \%$ to $25.3 \%$ while for MDF group the difference ranges from $3 \%$ to $11.2 \%$. The contribution of shear deformation of the core to the total deflection of large scale samples is also significant which ranges from $88 \%$ to $92 \%$ meaning that the 
Table 2. Theoretical and Experimental Deflection Values of Large Scale Sandwich Panels

\begin{tabular}{|c|c|c|c|c|c|c|c|c|c|c|c|}
\hline \multirow{2}{*}{\multicolumn{2}{|c|}{ Samples }} & \multicolumn{2}{|c|}{ Geometric } & \multirow[b]{2}{*}{$(\mathrm{EI})_{\mathrm{eq}}$} & \multirow[b]{2}{*}{$\mathrm{P}$} & \multirow[b]{2}{*}{$\delta_{\mathrm{b}}$} & \multirow[b]{2}{*}{$\delta_{\mathrm{s}}$} & \multirow[b]{2}{*}{$\delta_{\text {theo }}$} & \multirow[b]{2}{*}{$\delta_{\exp }$} & \multirow{2}{*}{$\frac{\delta_{\text {theo }}}{\delta_{\text {exp }}}$} & \multirow[b]{2}{*}{$\%$} \\
\hline & & $\mathrm{b}$ & $t_{c}$ & & & & & & & & \\
\hline \multirow{4}{*}{ CTR } & \multirow{2}{*}{2} & 101.2 & 51.90 & 9666786849 & 50 & 0.07 & 0.53 & 0.60 & 0.55 & 1.09 & 8.9 \\
\hline & & 101.2 & 51.90 & 9666786849 & 100 & 0.13 & 1.06 & 1.20 & 0.95 & 1.26 & 26.0 \\
\hline & \multirow{2}{*}{4} & 99.5 & 51.17 & 9243807464 & 50 & 0.07 & 0.55 & 0.62 & 0.55 & 1.12 & 12.5 \\
\hline & & 99.5 & 51.17 & 9243807464 & 100 & 0.14 & 1.10 & 1.24 & 1.1 & 1.12 & 12.5 \\
\hline \multirow{4}{*}{ JFC } & \multirow{2}{*}{3} & 100.7 & 40.23 & 11370452155 & 50 & 0.06 & 0.69 & 0.75 & 0.8 & 0.93 & -6.7 \\
\hline & & 100.7 & 40.23 & 11370452155 & 100 & 0.11 & 1.38 & 1.49 & 2.0 & 0.75 & -25.3 \\
\hline & \multirow{2}{*}{4} & 100.3 & 40.17 & 11298051840 & 50 & 0.06 & 0.69 & 0.75 & 0.8 & 0.94 & -6.2 \\
\hline & & 100.3 & 40.17 & 11298051840 & 100 & 0.11 & 1.39 & 1.50 & 2.0 & 0.75 & -24.9 \\
\hline \multirow{4}{*}{$\mathrm{MDF}$} & \multirow{2}{*}{3} & 99.63 & 39.33 & 9908906154 & 50 & 0.07 & 0.71 & 0.78 & 0.7 & 1.11 & 11.2 \\
\hline & & 99.63 & 39.33 & 9908906154 & 100 & 0.13 & 1.43 & 1.56 & 1.5 & 1.04 & 3.7 \\
\hline & \multirow{2}{*}{5} & 100.7 & 39.20 & 9962759772 & 50 & 0.06 & 0.71 & 0.77 & 0.7 & 1.10 & 10.4 \\
\hline & & 100.7 & 39.20 & 9962759772 & 100 & 0.13 & 1.42 & 1.55 & 1.5 & 1.03 & 3.0 \\
\hline
\end{tabular}

contribution of bending was only about $8 \%$ to $12 \%$. Overall, the deflection of hybrid sandwich panel was slightly larger than those of conventional sandwich panel although they have higher equivalent bending stiffness.

\section{Conclusions}

The experimental study of hybrid sandwich panels with intermediate layer has been carried out under four-point static bending loads. Two main findings are outlined as follows. First, the proposed model for predicting the deflection of hybrid sandwich panels provided fairly agreement results with the experimental values. The differences range from $3.9 \%$ to $35.4 \%$. Most of the sandwich panels showed experimental values lower than the theoretical values that can be considered as highly desirable in the design. Second, the introduction of intermediate layer does not contribute much to reduce the deflection of sandwich panel as the main contributor for the total deflection was the shear deformation of the core that mostly determined by the geometric of the samples and the thickness of the core.

\section{Acknowledgements}

The authors would like to acknowledge The Centre of Excellence in Engineered Fibre Composites (CEEFC), University of Southern Queensland (USQ) for providing access to laboratory facilities. Also, the authors would like to greatly appreciate the sponsorship from the Government of Indonesia through DGHE Project and The University of Mataram, which made this research possible.

\section{References}

1. Mamalis, A.G., Spentzas, K.N., Pantelelis, N.G., Manolakos, D.E., and Ionnidis, M.B., A New Hybrid Concept for Sandwich Structures, Composite Structures, 83, 2008, pp. 335-340.
2. Fajrin, J., Zhuge, Y., Bullen, F., and Wang, H., The Implementation of Statistical Inference to Study the Bending Strength of Sustainable Hybrid Sandwich Panel, Advanced Material Research, 250-253, 2011, pp. 956-961.

3. Fajrin, J., Zhuge ,Y., Bullen, F., and Wang, H., Significance Analysis of Flexural Behaviour of Hybrid Sandwich Panels, Open Journal of Civil Engineering, 3, 2013, pp. 1-7.

4. Fajrin, J., Alternative Theoretical Frameworks for Hybrid Sandwich Panel with Intermediate Layer, Jurnal Rekayasa Sipil, 11(2), 2015, pp 111.

5. Fajrin, J., The Application of Statistical Design of Experiments to Study The In-Plane Shear Behaviour of Hybrid Composite Sandwich Panel, Civil Engineering Dimension, 18(1), 2016, pp 25-30.

6. Davies, J.M., Lightweight Sandwich Construction, Blackwell Science, 2001, London.

7. Zenkert, D., An Introduction to Sandwich Construction, Solihull, EMAS, 1995.

8. Deshpande, V.S., The Design of Sandwich Panels with Foam Cores, Lecturing Handout, Cambridge University, 2002.

9. ASTM Standard C 393-00, Standard Test Method for Flexural Properties of Sandwich Construction, ASTM International, 2000, Philadelphia, Pa 19103.

10. Roylance, D., Beam Displacements, Department of Materials Science and Engineering, Massachusetts Institute of Technology, 2000, Cambridge, USA.

11. Manalo, A., Aravinthan, T., Karunasena, W., and Islam M.M., Flexural Behavior of Structural Fiber Composite Sandwich Beams in Flatwise and Edgewise Positions, Composite Structures, 92 (4), 2010, pp. 984-995.

12. Somayaji, S., Civil Engineering Materials, Prentice Hall, 1995, Englewood, New Jersey, USA. 
13. Teles, R.F., Menezzi, C.H.S., Souza, F., and Souza M.R., Theoretical and Experimental Deflections of Glued Laminated Timber Beams Made from Tropical Hardwood, Wood Material Science and Engineering, 2012, pp. 1-6.
14. Sharaf, T., Shawkat, W., and Fam, A., Structural Performance of Sandwich Wall Panels with Different Foam Core Densities in One-Way Bending, Journal of Composite Materials, 44 (19), 2010, pp. 2249-2263. 